Ann. Génét. Sél. anim., I980, 12 (4), 377-38r.

\title{
Influence d'une sélection sur le comportement sur la réponse à l'infection coccidienne chez la poule
}

\author{
J. M. FAURE $\left({ }^{*}\right)$ et P. YVORÉ $\left({ }^{* *}\right)$ \\ (*) Domaine expérimental du Magneraud, I.N.R.A., \\ I770o, Surgères, France. \\ (**) Station de Pathologie aviaive et de Parasitologie (*), I.N.R.A., \\ Centre de Recherches de Tours-Nouzilly, \\ 37380 Monnaie, France
}

\begin{abstract}
Résumé
La sélection en open-field permet d'obtenir des souches de poulets dont l'émotivité est différente. La réponse à une infection expérimentale par Eimeria tenella est plus importante chez la souche " active " que chez la souche "inactive".
\end{abstract}

En I931, Kralingaer et Chodziesner ont mis en évidence les différences de sensibilité à l'infection coccidienne entre volailles de races Leghorn, Faverolles et leurs croisements. Si la possibilité d'une sélection vis-à-vis de la coccidiose caecale à E. tenella a été démontrée (MAYGEW, I934; HERRICK et al., I935), différents travaux permettent de penser que la résistance obtenue n'est que partielle (ROSENBERG et al., I954; Champion, I954). Plus récemment, JEFFERS et al. (1970) ont constaté avec $E$. tenella qu'une sélection sur le critère mortalité ne permettait pas d'obtenir des individus plus résistants au parasitisme.

L'importance de l'environnement et des facteurs d'agression dans l'étiopathogénie des coccidioses est maintenant reconnue. Cela a incité Gross (I976) à comparer la réponse à une infection par $E$. necatrix de deux souches de poulets ayant des taux de corticostérone plasmatique différents et soumises à des niveaux de "stress " différents. L'auteur a constaté des différences dans l'importance des lésions et dans l'incidence du parasitisme sur le gain de poids.

FAURE (I975) a montré que la sélection pour l'activité en "open-field" entraînait une diminution de l'émotivité de la souche active par rapport à la souche inactive. En outre, ces souches diffèrent aussi par leur taux de corticostérone plasmatique (RoMBaUTS et FAURE, résultats non publiés). Il nous a paru intéressant d'étudier la sensibilité des souches obtenues à l'infection par E. tenella. 


\section{Matériel et méthodes}

Les animaux proviennent d'une souche Cornish sélectionnée de manière divergente pour l'activité en "open-field " à l'âge de 2 jours pendant 7 générations (FAURE et Folmer, I975). 97 animaux de souche "active " et I27 de souche " inactive " ont été utilisés.

Ces animaux ont été élevés au sol et contaminés pendant 5 jours consécutifs à partir de l'âge de $\mathrm{I} 2$ jours par administration d'un aliment contaminé artificiellement par des oocystes d'E. tenella (Yvorí et al., I980). Il's ont reçu en moyenne 5000 oocystes sporulés /animal/jour.

Quinze animaux de chaque souche ont été prélevés au hasard 9 jours après le début de l'infection dans le but d'évaluer l'incidence du parasitisme à ce moment. Ils ont été pesés. On a mesuré le taux d'éléments figurés du sang par microhématocrites et la coloration sérique par lecture directe au spectrophotomètre à $480 \mathrm{~nm}$ contre de l'eau distillée.

Ces deux tests permettent une mesure assez fidèle de l'importance de l'infection. E. tenella provoque des hémorragies qui diminuent la valeur de 1'hématocrite. La perte sérique et le défaut d'apport de pigments caroténoïdes dû à la sous-consommation alimentaire sont à l'origine d'une décoloration sérique en rapport avec l'importance de l'infection (YvORE, I978). Enfin, les lésions caecales ont été évaluées, cette évaluation se limitant cependant à l'observation de leur présence ou de leur absence sans notation de leur degré d'importance.

La croissance des animaux des deux souches a été mesurée par pesées individuelles à 9 jours (avant l'infection), 22 jours ( 9 jours après le début de l'infection), 29 jours ( 15 jours après le début de l'infection) et à 36 jours (2I jours après le début de l'infection).

Les morts ont été comptés et autopsiés jour par jour.

Les gains de poids des animaux ont été comparés aux différents âges par une analyse de variance à deux facteurs (souche, sexe et interaction). Les résultats d'hématocrites et de colorations sériques ont été comparés en employant le test $U$ de Mann-Whitney. Un test de Fisher (test du binome) (SIEGri, I956) a servi à analyser la présence ou l'absence de lésions caecales.

\section{Résultats}

\section{I. - Mortalité}

Aucun animal n'est mort de coccidiose. On pouvait s'attendre à ce résultat compte tenu d'une infection volontairement modérée des animaux.

\section{2. - Croissance pondérale}

Les différences de poids dues à la souche sont significatives lors de la pesée effectuée environ 15 jours après l'infection des animaux. Les animaux "actifs" ont un poids inférieur à celui des animaux " inactifs" (tabl. I et 2). 


\section{TABIEAU I}

Poids moyen des animaux dans les lots "Actifs" $(A)$ et "Inactifs" $(I)$ au cours de l'élevage

Average weight of animals in "Active" $(A)$ and "Inactive" $(I)$ lots during breeding

Ages des animaux (1)

\begin{tabular}{|c|c|c|c|c|}
\hline & J. I 2 & J. $2 \mathrm{I}$ & J. 28 & J. 35 \\
\hline 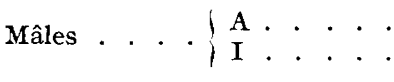 & $\begin{array}{l}\mathrm{I} 40,3 \\
\mathrm{I} 36, \mathrm{I}\end{array}$ & $\begin{array}{ll}302,2 & a \\
301,6 & a\end{array}$ & $\begin{array}{ll}46 \mathrm{r}, 4 & a \\
48 \mathrm{r}, \mathrm{I} & b\end{array}$ & $\begin{array}{l}652,7 \text { a } \\
649,9 a\end{array}$ \\
\hline Femelles. . $\left\{\begin{array}{llll}\mathrm{A} & \cdot & \cdot & \cdot \\
\mathrm{I} & \cdot & \cdot & \cdot\end{array}\right.$. & $\begin{array}{l}\mathrm{I} 30,4 a \\
\mathrm{I} 29, \mathrm{O}\end{array}$ & $\begin{array}{l}277,0 \quad a \\
280,8 \text { a }\end{array}$ & 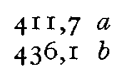 & $\begin{array}{ll}58 \mathrm{o}, \mathrm{o} & a \\
57 \mathrm{I}, \mathrm{I} & a\end{array}$ \\
\hline
\end{tabular}

(1) Début de l'infection à $\mathrm{J}$. I2.

Pour un même jour et un même sexe, deux valeurs suivies de lettres différentes sont significativement différentes : $\mathbf{P}<0,05$.

TABLEAU 2

Poids corporels: valeurs de $F$ (analyses de variance à 2 facteurs) du sexe, de la souche et interactions; effets à differents âges

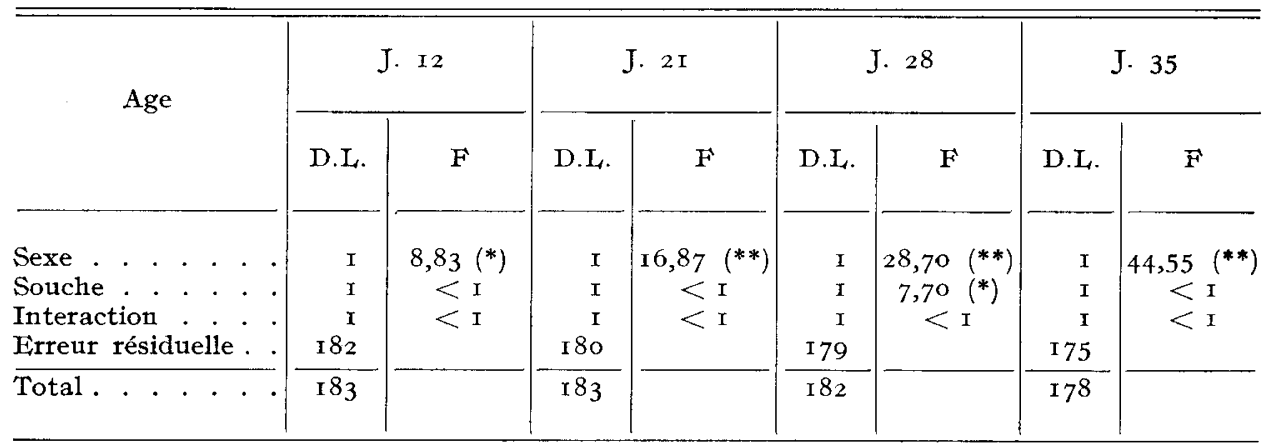

D.I. : degré de liberté.

$\left(^{*}\right): \mathrm{P}<\mathrm{O}, \mathrm{OI}$

$(* *): \mathrm{P}<0$,oor.

\section{3. - Évaluation de l'infection des animaux}

Au tableau 3, nous avons rassemblé les résultats obtenus sur les I5 animaux de chaque souche prélevés 9 jours après le début de l'infection.

Les lésions sont moins fréquentes dans le lot "Inactifs". La différence est 
TABLEAU 3

Sevérité de l'infection dans les souches " actives " et "inactives" $(\mathrm{n}+15)$ 9 jours après l'infection

Severity of the infection in the "active" and "inactive" strains ( $\left.\mathrm{n}+\mathrm{I}_{5}\right)$ 9 days after the infection

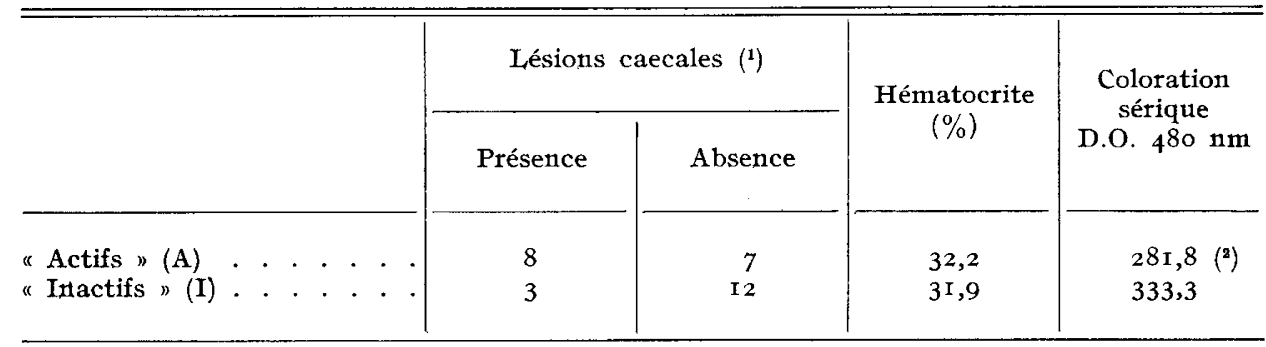

( ) Test de Fisher (test du binome) $\mathbf{P}<0,05$ entre " actifs " et "inactifs".

(2) Valeurs moyennes des lignées A et I significativement différentes $(\mathbf{P}<0,05)$ test de Mann-Whitney

au seuil de signification de $5 \mathrm{p}$. Ioo, ce qui est à notre avis convenable compte tenu du faible nombre d'animaux prélevés.

$\mathrm{Si}$ on ne constate pas de différence pour les valeurs de l'hématocrite, on note une coloration plus faible du sérum chez les animaux actifs $(P<0,05)$.

\section{Discussion}

L'infection n'a pas entrainé de maladie grave et n'est à l'origine d'aucune mortalité. Cela a été volontairement recherché en administrant aux animaux un faible nombre d'oocystes. Il nous a en effet semblé préférable d'éviter un phénomène de tout ou rien, la mortalité, difficilement analysable et qui ne correspond pas à ce que l'on observe en élevage où l'infection coccidienne se manifeste plutôt par une action sur les performances des animaux.

Malgré la discrétion des manifestations et un nombre réduit d'animaux en expérimentation, nous avons pu mettre en évidence des réponses différentes des deux souches à l'infection. Pour tous les critères, à l'exception de l'hématocrite, les " actifs" se sont révélés plus sensibles que les "inactifs".

Nos résultats avec E. tenella sont en accord avec ceux de Gross (1976) qui utilisait E. necatrix. Cet auteur trouve en effet qu'une lignée à haut taux de corticostérone est plus résistante qu'une lignée à faible taux et que l'élevage dans des conditions stressantes augmente la résistance.

La sélection pour un comportement se révèle donc intéressante dans la recherche d'une résistance à certains agents pathogènes et mériterait d'être mieux étudiée.

Reçu pour publication en janvier $198 \pi$.

\section{Summary}

\section{Influence of a selection on behaviour on response to coccidian infection in the Hen}

A strain selected for high open-field activity shows lower emotionality and lower levels of corticosterone than a strain selected for low activity. An experimental infection with Eimeria tenella has more effect on the active than the inactive strain. 


\section{Références bibliographiques}

Champion L. R., 1954. The inheritance of resistance to cecal coccidiosis in the domestic fowl. Poult. Sci., 33, 670-681.

Faure J. M., I975. Étude des liaisons entre comportement en "open-field " et émotivité chez le jeune poussin. Ann. Génét. Sél. anim., 7, 197-204.

FaUre J. M., Folmer J. C., I975. Étude génétique de l'activité précoce en " open-field" du jeune poussin. Ann. Génét. Sél. anim., 7, I23-132.

Gross W. B., I976. Plasma steroid tendency, social environment and Eimeria necatrix infections. Poult. Sci., 55, I508-1512.

HERRICK C. A., OTT G. I., HELPIN J. G., Holmes C. E., I935. Extra calcium in ration increases susceptibility of chicks to coccidiosis. Wisconsin Agricultural Experimental Station Bulletin, 430, $97-98$.

JeFFERS T. K., Challey J. R., McGrbBon W. H., I97o. Response of several lines of fowl and their single-cross progeny to experimental infection with Eimeria tenella. Avian Disease, 14, 203-2I0.

KRALLINGer H. G., ChODzIESNER M., I93I. Eine Studie über die Kokrichen-resistenz der Faverolle-Leghorn Kreuzungen. Landwirtschaftliche Jahrbüchur, 74, 697-7 I I.

MAYGEW R. I., I934. Studies on coccidiosis. VIII. Immunity or resistance to infection in chickens. Journal of the Veterinary Medical Assoc., 85, 729-734.

RosenberG M. M., Alicata J. E., Palafox A. L., I954. Further eveidence of hereditary resistance and susceptibility to cecal coccidiosis in chickens. Poult. Sci., 33, 972-980.

SIEGEL S., 1956. Non parametric statistics for the hebavioral science. Mac Graw Hill, New York. YvORE P., 1978. Effect of coccidiosis on the nutrition of the host. Avian coccidiosis pp. 26928o. Ed. P. L. Long, K. N. Boorman et B. M. Freeman. Brit. Poult. Sci., Ld Edinburg.

Yvore P., Raynaud J. P., Conan L., Naciri M., Virat M., I980. Method of evaluating the efficiency of anticoccidial drugs in floor-pen tirals with multiple in-feed infection versus "Seeding" model. Ann. Rech. Vet., 11 (I I), sous presse. 